\title{
WIND ENERGY CONVERSION GENERATOR
}

\section{Vitalijs Beresnevich, Shravan Koundinya Vutukuru, Martins Irbe, Edgars Kovals, Maris Eiduks, Kaspars Burbeckis, Janis Viba}

Riga Technical University, Latvia

vitalijs.beresnevics@rtu.lv, vshravankoundinya1989@gmail.com, martins.irbe@rtu.lv, edgars.kovals@gmail.com, maris.eiduks@rtu.lv, kaspars.burbeckis@temeso.lv, janis.viba@rtu.lv

\begin{abstract}
The aim of the paper is to analyze and optimize the operational safety and efficiency of wind energy conversion equipment. A wind energy conversion device equipped with one oscillating flat blade has been developed and studied. In this device, lateral surface of the blade is firmly attached to the crank, which is kinematically connected with the generator's slider moving inside the electric coil. As a result, electrical energy is produced in the linear generator. The considered electromechanical device is described by the second order differential equation. In this equation, the interaction of wind flow and relative motion of the blade is described by the approximate relationships of classical mechanics. Operation of the system due to the action of air flow is simulated with computer program Mathcad. Possibilities to obtain energy with generators of different characteristics, the operation of which is regulated by mechatronic control, have been studied. The effect of a constant wind flow with a constant speed and also with a harmonic or poly harmonic component is considered. Partial parametric optimization of the electromechanical system has been performed. Graphs for the change of plate phase coordinates, as well as for the change of the obtained power are presented. The results obtained in the paper can be used in the study of similar fluid flow interactions at damping or energy extraction.
\end{abstract}

Keywords: air flow, oscillating blade, energy conversion, generator.

\section{Introduction}

Various types of devices and mechanisms are used for energy extraction from air flow. Usually, most of the energy scavenging or energy extraction devices possess multiple blades that are oriented at an angle to the air or fluid flow. The main principle of their operation includes air flow action on blades mounted on a special wheel and further transformation of air flow kinetic energy into the mechanical energy of wheel rotation $[1 ; 2]$. The present device involves only a single flat blade that is subjected to air flow. The flat plate is connected to a slider of a linear generator with the help of a connecting rod for the prime purpose of power generation. However, this equipment does not provide a possibility for special change of the blade's orientation relative to the air flow during rotation of the main wheel, and therefore position of the blade can be optimal only in specific time instants [3].

Theoretical analysis of the air flow interaction with the rotating flat blade in different aerodynamic conditions is considered in [4]. Special variation of the flat blade's turning angle during rotation of the main wheel is realized in the devices described in $[5 ; 6]$. But these devices have drawbacks, which are especially manifested with an increase in the number of flat blades and existing trend for $t$ increasing of the blade's number is caused by the desire to give raise for the efficiency of wind energy conversion and for the generated power. The effect of the number of blades on the maximum power co-efficient was studied, and it was found that the turbine with 3 blade configuration was more efficient than 5 or 6 blade turbine configuration [7]. Actually, in the case of the number of blades more than one, where a comparison between 2 and 3 bladed wind turbines and their effect on turbulent wake behind the turbine was studied, it was concluded that 2-bladed turbine had higher wake recovery, which, in turn, relates to higher power density per unit land area [8]. Therefore, for more numbers of blades, that is, blade more than one, the front (from the side of the air flow) blades clog and interfere with the access of the air flow to the rear blades. As a result, the air flow due to the rear blades may vary in direction and size, with air vortexes forming between the blades. In general, it breaks the rotation of the central rotor, which negatively affects the efficiency of wind energy conversion. Besides, the rise of the operational efficiency of existing devices can be achieved by increasing of radial dimensions of rotating blades. But this approach has a negative influence on reliability of wind equipment due to increase in the blade's end speed and high generated noise. As a result, the probability of traumatizing birds and other living beings with a rotating flat blade increases.

This paper deals with the development of a new method for wind energy conversion based on utilization of flat blade translation motion. 


\section{Model of wind energy conversion generator}

The model of wind energy conversion device considered in this paper is shown in Figure 1. The device contains a flat blade 1 , attached to the rotating axle 2 by a cylindrical axial pivot. Longitudinal axis of the rotating axle 2 coincides with the symmetry axis $z_{1}$ of the flat blade 1 and is firmly connected to the slider 3, which has the ability of translation motion along special guides in the direction of the $x$ axis. In addition, the sliding motion is limited by springs 4 and shock absorbers 5 . Besides, the rotation of the oscillating blade 1 around its axis of symmetry $z_{1}$ is limited by a rotational spring 6 and a shock absorber 7. Perpendicular to the lateral surface of the flat blade 1, a crank 8 is firmly attached, and the second end of the crank 8 is hinged to the connecting rod 9 . Besides, the rod 9 is hinged to the electric generator slider 10 that has the ability to move inside the electric coil 11 in the direction of the $x_{1}$ axis.

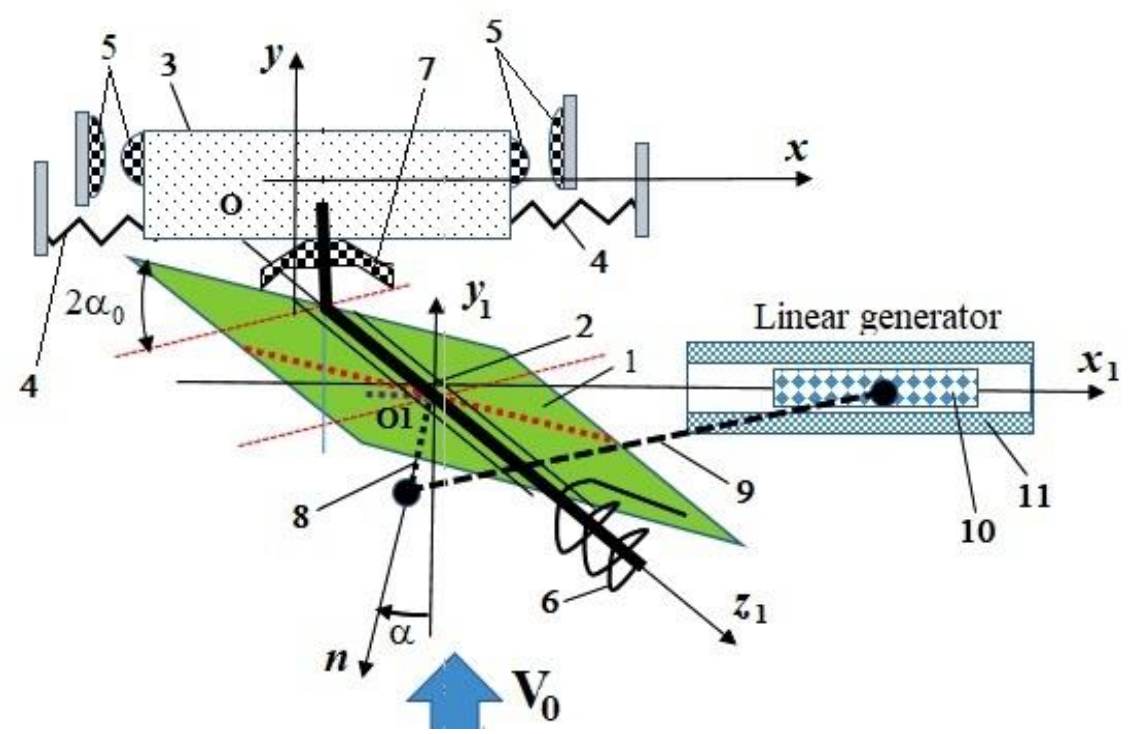

Fig. 1. Principle model of the wind energy conversion generator: 1 - flat blade; 2 - rotating axle; 3 - slider; 4 - spring; 5 - shock absorber; 6 - rotational spring; 7 - rotational shock absorber; 8 - crank; 9 - connecting rod; 10 - slider of linear generator; 11 - electric coil

Operation of the developed wind energy conversion device is as follows. Cyclic motion of the device begins in its position shown in Figure 1. The wind flow at a speed of $\mathrm{V}_{0}$, directed perpendicular to the direction $x$ of motion of the slider 3 , acts on the flat blade 1 . In the direction of normal $n$, a force $N$ is formed, which causes the slider 3 to move along $x$ axis to the right (Fig. 1-2). As a result, compressive force $F_{k}$ is formed in the connecting rod 9 . The compressive force $F_{k}$ from the connecting rod 9 and the braking linear generator consisting of a slider 10 and an electric coil 11 keeps the rotating flat blade 1 near the left rotational shock absorber 7. In this position of the device, the turning angle $\alpha$ reaches its maximum value $\alpha_{0}$ in clockwise direction. The spring 4 is stretched, then the deformation of the right shock absorber 5 occurs until the slider 3 stops at the end right position.

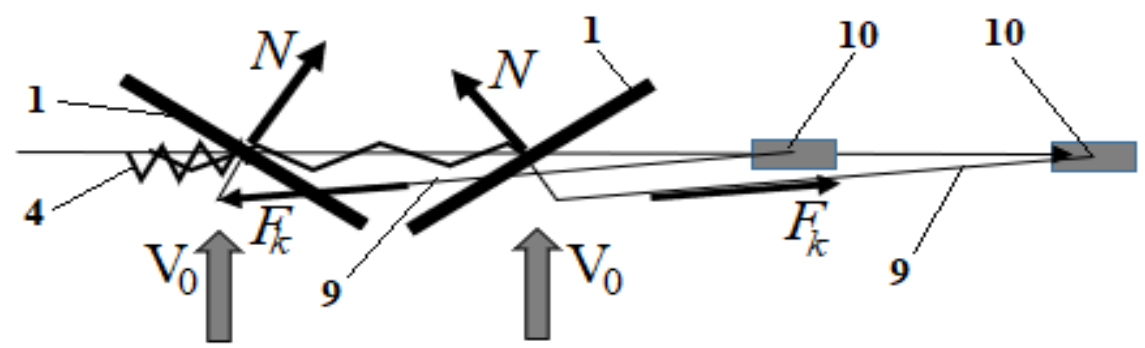

Fig. 2. Sequential positions of blade during operation of the wind device: 1 - flat blade; 4 - spring; 9 - connecting rod; 10 - slider of linear generator

Then, as a result of the action of elastic forces from the spring 4 and the right shock absorber 5 , the slider 3 moves back to the left. At the beginning of such a movement, the connecting rod 9 is tensioned, and, consequently, the direction of force $F_{k}$ changes to the opposite (Fig. 1-2). Flat blade 1 turns around 
symmetry axis $z_{1}$ counterclockwise until reaching the right rotational shock absorber 7 . In this position of the device, the turning angle $\alpha$ reaches its maximum value $\alpha_{0}$ in counter clockwise direction. As a result, the position of normal $n$ to flat blade is changed against the wind flow $V_{0}$. Now the force $F_{k}$ presses on the flat blade 1 in the direction opposite to the $x$ axis. As a result, the slider 3 , moving to the left, sequentially deforms the spring 4 and the left shock absorber 5 until the slider 3 stops at the end left position. The cycle will then repeat as the compressive force $F_{k}$ will re-emerge in the connecting rod 9.

During this cyclic movement, the slider 10 of the linear generator moves backward inside the electric coil 11 along the $x_{1}$ axis. As a result, electrical energy is produced in the linear generator (alternating current is generated in the electric coil 11). The principle of operation of a linear generator is described in more detail in the literature [9].

\section{Simulation of flat blade motion}

A two degree of freedom (2DOF) model for a translation motion $x$ and rotation $\varphi$ motion of thin flat plate (thickness $\mathrm{d} \sim 0$ ) is shown in Figure 3. In the model, for translation motion a linear elastic element is included with stiffness coefficient $c_{1}$ and for rotation motion elastic element with stiffness coefficient $c_{2}$. Air flow with velocity $V_{0}$ is normal to $x$-axis. The motion of the given model for long crank is described by the following two differential equations (1) and (2):

$$
\begin{gathered}
\left(m_{1}+m_{2}\right) \cdot \ddot{x}=-c_{1} \cdot x-b \cdot[\dot{x}+r \cdot \dot{x} \cdot \sin (\varphi)]+ \\
-(1+C) \cdot L \cdot B \cdot \rho \cdot\left[V_{0} \cdot \cos (\varphi)+\dot{x}\right]^{2} \cdot \operatorname{sign}\left[V_{0} \cdot \cos (\varphi)+\dot{x}\right] \cdot \sin (\varphi) ; \\
J_{c} \cdot \ddot{\varphi}=-c_{2} \cdot \varphi-b_{2} \cdot \dot{\varphi}+b \cdot[\dot{x}+r \cdot \dot{x} \cdot \sin (\varphi)] \cdot r \cdot \sin (\varphi),
\end{gathered}
$$

where $m_{1}, m_{2}-$ masses of bodies;

$J_{c}$ - moment inertia of rotating mass against the central axis;

$\ddot{x}, \dot{x}, x$ - linear acceleration, velocity and displacement;

$\ddot{\varphi}, \dot{\varphi}, \varphi-$ angular acceleration, velocity and displacement;

$L, B$ - length and width of the plate;

$c_{1}, c_{2}, b, b_{2}$ - parameters of the system;

$C$ - constant, that for the long plate $C=0.5[6]$;

$V_{0}$ - flow velocity; $\rho$ is the density of the fluid;

$r$ - length of the crank 8 (Fig. 1.).

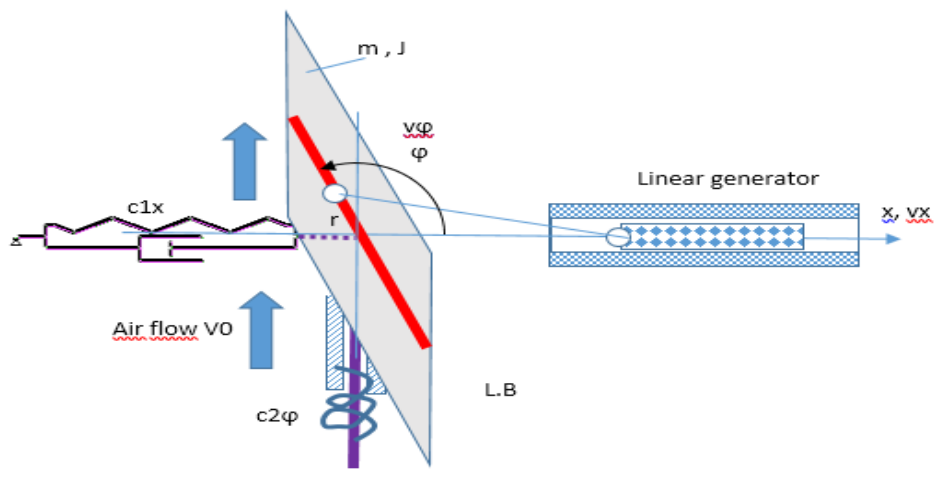

Fig. 3. Mathematical model of thin flat plate interacting with air flow

The renewable energy is generated due to the action of the damping force $\{b \cdot[\dot{x}+r \cdot \dot{x} \cdot \sin (\varphi)]\}$. Therefore, momentary power can be determined by formula

$$
P=b \cdot[\dot{x}+r \cdot \dot{x} \cdot \sin (\varphi)]^{2} .
$$

The average power $P_{a}$ during time $t$ is determined by integration of equation (3):

$$
P a=\frac{\int_{0}^{t} b \cdot[\dot{x}+r \cdot \dot{x} \cdot \sin (\varphi)]^{2} \cdot d t}{t} .
$$


By the analysis of equations (1) and (2), it can be concluded that minimum nine parameters can be used to control the efficiency of this system. These parameters are as follows: $\mathrm{m}=m 1+m 2, J c, c 1$, $c 2, b, b 2, r, A=L \cdot B$ and $V 0$.

Mathematical simulation of equations (1) and (2) was performed with program MathCad, assuming the following values of main system parameters: $A=0.04 \mathrm{~m}^{2} ; V_{0}=10 \mathrm{~m} \cdot \mathrm{s}^{-1} ; \rho=1.25 \mathrm{~kg} \cdot \mathrm{m}^{-3}$.

Results of simulation of the plate motion are presented in Figs. 4 -7. As it is seen from the analysis of the graphs presented, almost stationary oscillatory regime with maximal average power $P_{a}$ can be achieved after some cycles of the transient process.

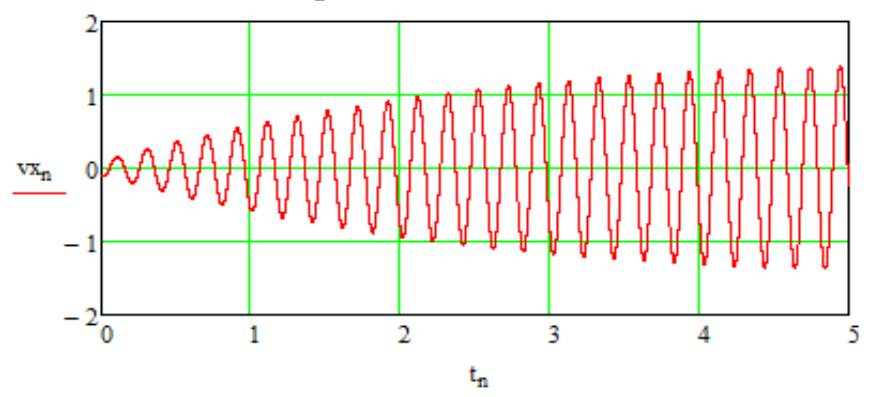

Fig. 4. Velocity $v$ of plate central point as function of time $t$

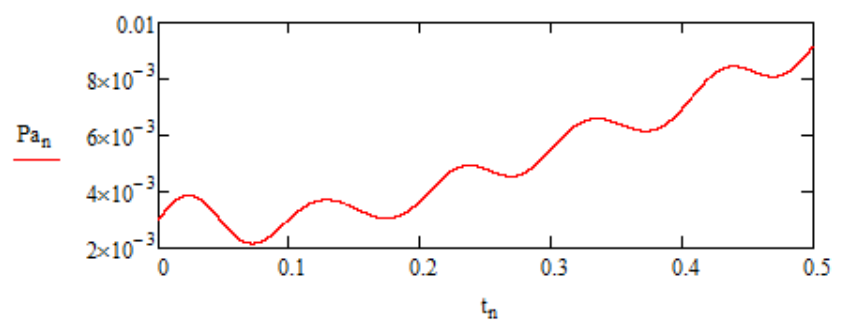

Fig. 5. Average power $\boldsymbol{P}_{a}$ of generator force $\boldsymbol{b} \dot{\boldsymbol{x}}$ during long transient process $(t=30 \mathrm{~s})$

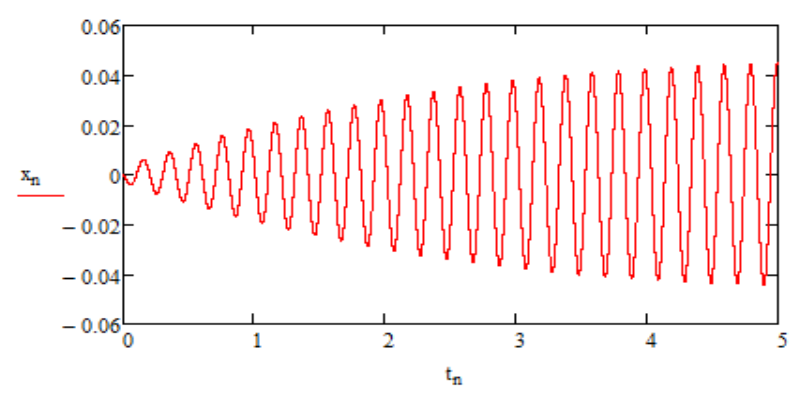

Fig. 6. Displacement $x$ of plate as function of time $t$

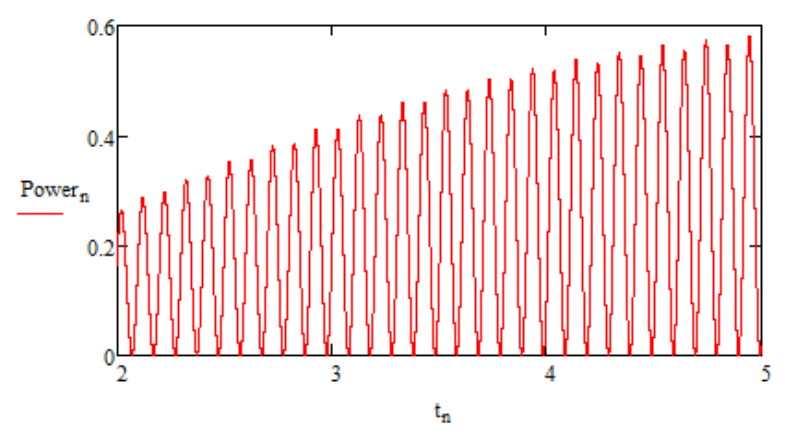

Fig. 7. Cyclic change in time of generator power $\boldsymbol{P}_{a}$ 


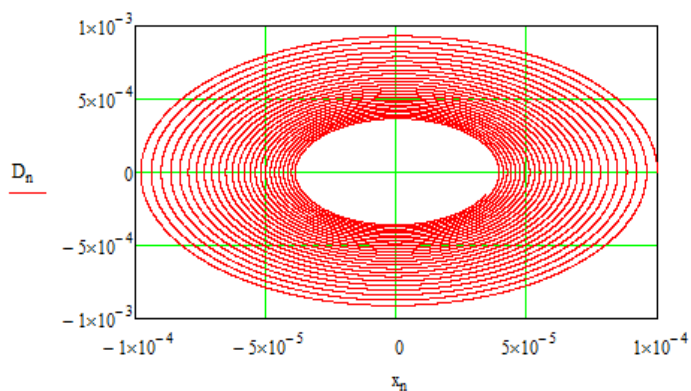

Fig. 8. Generator force $D=b \cdot[\dot{x}+r \cdot \dot{x} \cdot \sin (\varphi)]^{2}$ as function of displacement

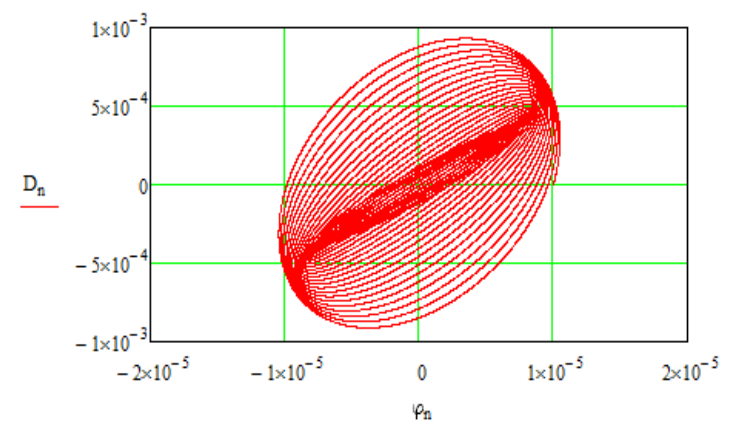

Fig. 9. Generator force $D=b \cdot[\dot{x}+r \cdot \dot{x} \cdot \sin (\varphi)]^{2}$ as function of rotation angle

Computer modeling showed that the nine parameters of the system need to be optimized to obtain the maximum power. For full analysis of the proposed mechanism, linear elasticity and the use of linear dampers (generators) alone are not enough. This means that with the introduction of nonlinear suspension, the effects of nonlinear dynamics, including mode bifurcations, will appear. Such issues have been studied, but their results are not included in this work due to their large volume.

\section{Results and discussion}

The proposed model of wind energy conversion generator (Fig. 1) has some advantages over the known devices [1-6] due to its specific design. The proposed model has one flat blade 1, so wind flow twirls between the blades are impossible in principle, but such twirls reduce the efficiency and safety of the known equipment. In addition, generation of useful power in the proposed device is due to the translation movement of the flat blade 1 and the slider 3 (Fig. 1), so the wind flow load is uniformly distributed over the lateral surface of the flat blade 1 . This provides a simple way to increase the operational efficiency of the developed device. This can be achieved by increasing the area $A$ of the lateral surface of the flat blade 1 , since in proportion to the area $A$, the generating power $P$ increases according to the formula (5):

$$
P=C_{0} \cdot A \cdot V^{3},
$$

where $C_{0}$ - co-efficient;

$V$ - relative velocity of wind flow to normal surface of flat blade 1 (Fig. 1).

Only the linear energy generator is considered here in the current work with partial parametric optimization of the electromechanical system. It would be interesting to know the shape effects of the flat blade (like bent, curved and twisted) on the operational efficiency of the device. The mathematical simulations for the change in flow velocity as harmonic or poly-harmonic time functions pave a way for realizing better efficiency of the system. However, to validate the results of this article with the existing literature the task of optimization is a must.

\section{Conclusions}

1. The wind flow load is analysed to be uniformly distributed on the lateral surface of the blade, a condition for which good operational efficiency of the device is supposed to be achieved.

2. In the process of obtaining good energy efficiency of the device not only a constant flow velocity environment is to be analysed, but it is equally essential to check for unsteady and gusty flow conditions.

3. Though the viscous nature of the fluid medium is ignored, the mathematical simulations show reasonable and acceptable device behaviour with appropriate power generation results.

4. The shape effects, parameters of the system and nature of flow (steady, unsteady etc) are of primary importance when considering the simple task of synthesis and optimization in the air medium.

5. Only a partial parametric optimization task is taken up in the current work for overall better efficiency other parameters of the system to be optimized. 


\section{Acknowledgements}

The article is formed from a patent application submitted in Latvia by Riga Technical University.

\section{References}

[1] Wind power: recent developments/edited by D.J. de Renzo. Park Ridge, New Jersey, USA: Noyes Data Corporation, 1979. 347 p.

[2] Dirba J., Levins N., Pugačevs V. Vēja enerǵijas elektromehāniskie pārveidotāji (Electromechanical converters of wind energy). Riga: RTU Publishing House, 2006. 300 p. (In Latvian).

[3] Янсон P.A. Ветроустановки (Wind installations). Moscow: Publishing House of N.Bauman MSTU, 2007. 36 p. (In Russian).

[4] Viba J, Beresnevich V, Noskovs S, Irbe M. Investigation of rotating blade for energy extraction from fluid flow. Vibroengineering Procedia, vol. 8. Kaunas: JVE International Ltd.; 2016, pp. 312315.

[5] Viba J., Beresnevich V., Irbe M., Dobelis J. The control of blades orientation to air flow in wind energetic device. Energy Procedia, 2017, No 128, pp. 302-308.

[6] Viba J., Beresnevich V., Irbe M. Synthesis and Optimization of Wind Energy Conversion Devices. Chapter in a book: Design Optimization of Wind Energy Conversion Systems with Applications. IntechOpen, London, 2020, pp. 125-141.

[7] Eltayesh A., Castellani F., Burlando M., Hanna M.B., Huzayyin A.S., El-Batsh H.M. and Becchetti M. Experimental and numerical investigation of the effect of blade number on the aerodynamic performance of a small-scale horizontal axis wind turbine. Alexandria Engineering Journal, 2021, Vol. 60. pp. 3931-3944.

[8] Muhle F., Adaramola M.S. and Saetran L. The effect of the number of blades on wind turbine wakea comparison between 2-and 3-bladed rotors. Journal of Physics, 2016.

[9] Баль В.Б., Геча В.Я., Гончаров В.И. и др. Линейные электрические машины возвратнопоступательного действия - области применения (Linear electric machines of reciprocating motion - fields of application). Problems of electromechanics, 2015, vol. 149, pp. 3-17. 